\title{
Development of stable reporter system cloning luxCDABE genes into chromosome of Salmonella enterica serotypes using Tn7 transposon
}

\author{
Kevin Howe ${ }^{1}$, Attila Karsi ${ }^{2,3}$, Pierre Germon ${ }^{4}$, Robert W Wills ${ }^{1}$, Mark L Lawrence ${ }^{2,3^{*}}$, Richard H Bailey ${ }^{1 *}$
}

\begin{abstract}
Background: Salmonellosis may be a food safety problem when raw food products are mishandled and not fully cooked. In previous work, we developed bioluminescent Salmonella enterica serotypes using a plasmid-based reporting system that can be used for real-time monitoring of the pathogen's growth on food products in short term studies. In this study, we report the use of a Tn7-based transposon system for subcloning of luxCDABE genes into the chromosome of eleven Salmonella enterica serotypes isolated from the broiler production continuum.

Results: We found that the lux operon is constitutively expressed from the chromosome post-transposition and the lux cassette is stable without external pressure, i.e. antibiotic selection, for all Salmonella enterica serotypes used. Bioluminescence expression is based on an active electron transport chain and is directly related with metabolic activity. This relationship was quantified by measuring bioluminescence against a temperature gradient in aqueous solution using a luminometer. In addition, bioluminescent monitoring of two serotypes confirmed that our chicken skin model has the potential to be used to evaluate pathogen mitigation strategies.

Conclusions: This study demonstrated that our new stable reporting system eliminates bioluminescence variation due to plasmid instability and provides a reliable real-time experimental system to study application of preventive measures for Salmonella on food products in real-time for both short and long term studies.
\end{abstract}

\section{Background}

Salmonella enterica is indigenous to the gastrointestinal tracts of many mammals, birds, and reptiles without the manifestation of adverse effects on the host. However, subclinical infections of Salmonella in animals have the potential to cause disease in humans exposed to food products that are mishandled during processing or inappropriately cooked $[1,2]$. Cross-contamination during the slaughter process contributes to the transmission of food borne pathogens and therefore increases the risk of disease in humans. Throughout the processing plant, opportunities arise for the spread of bacteria from contaminated carcasses to uncontaminated carcasses $[3,4]$.

\footnotetext{
*Correspondence: lawrence@cvm.msstate.edu; rhbailey@cvm.msstate.edu 'Department of Pathobiology and Population Medicine, College of Veterinary Medicine, Mississippi State University, Mississippi State, MS 39762, USA

${ }^{2}$ Department of Basic Sciences, College of Veterinary Medicine, Mississippi State University, Mississippi State, MS 39762, USA
}

Regardless of whether the source of contamination was pre-harvest or post-harvest, Salmonella is difficult to remove from carcasses due to its ability to adhere to chicken skin and endure the different stages of processing [5]. Laboratory research, as well as in-plant trials, has demonstrated this relationship [6-9]. Therefore, persistence of Salmonella within the processing plant may be partially explained by interactions between chicken skin and Salmonella [10]. Under controlled conditions, chemical treatments are effective in the reduction of Salmonella levels on broiler carcasses or skin [11-14]. However, gaps in the knowledge base exist relative to the persistence of Salmonella during processing and the most appropriate methods for reduction and control of the microorganism.

Bioluminescence imaging (BLI) is a technique that can be used for real-time quantification and tracking of live bacteria in hosts [15-18]. Previously, a BLI based realtime monitoring system for Salmonella enterica serotypes was developed by our group that employs the 
plasmid pAKlux1, which carries a bacterial luciferase gene isolated from Photorhabdus luminescens [19]. However, the use of this plasmid-based bioluminescence system requires continuous antibiotic selection during the course of experiments to prevent plasmid instability in Salmonella enterica serotypes [19], which may not be suitable for long-term in-vitro and in-vivo studies.

In response to this limitation, we now report cloning of the luxCDABE operon into a stable tn7-based transposon system that inserts the $\operatorname{lu} x C D A B E$ genes into a specific location in the Salmonella chromosome. We successfully used this transposon system to stably insert the bacterial lux operon into eleven Salmonella enterica serotypes isolated from the broiler production continuum, including post hatchery, prior to harvest, arrival at the plant, pre-chill tank, and post-chill tank. We also conducted a series of experiments to quantify bioluminescence expression in these Salmonella enterica isolates under environmental conditions that may be present in poultry processing. This reporter system can be applied in future research to further understand how Salmonella are able to persist throughout the poultry processing continuum, and similar situations pertinent to the food industry.

\section{Results and Discussion}

\section{Construction of plasmid pBEN276}

Plasmid pGRG25 features a site-specific recombination system based on the bacterial transposon $\operatorname{Tn} 7$ [20]. The Tn7 system inserts at the $\operatorname{att} \operatorname{Tn} 7$ site and is oriented specifically such that the right end of $\operatorname{Tn} 7$ is adjacent to the 3' end of the $\operatorname{glm} S$ gene [21], and it has been used for transgene insertion into the chromosome of Escherichia coli, Salmonella, and Shigella [20]. Plasmid pGRG25 is also a temperature-sensitive delivery plasmid that can be cured after transgene insertion at the attTn 7 site by culturing at $42^{\circ} \mathrm{C}$. Plasmid pBEN276 contains the luxCDABE operon between the Tn7 transposon arms on plasmid pGRG25, and its expression is driven by the E. coli frr promoter (Figure 1), which controls expression of a house-keeping gene encoding ribosome recycling factor. Thus the lux operon will be expressed constitutively. The chromosomal insertion point is specific and insertion of $l u x$ operon does not disrupt the function of $g \operatorname{lm} S$ gene, therefore it is highly unlikely that bacterial physiology will be affected adversely [20].

\section{Characterizing the bioluminescent properties of Salmonella enterica}

Plasmid pBEN276 was utilized to insert the bacterial lux operon into chromosomes of eleven Salmonella enterica serotypes. Bioluminescence correlated well to bacterial population density in all serotypes used, as exemplified in $S$. Montevideo ( $\mathrm{p}=<0.0001, \mathrm{r}^{2} 0.94$ ) (Figure 2). The

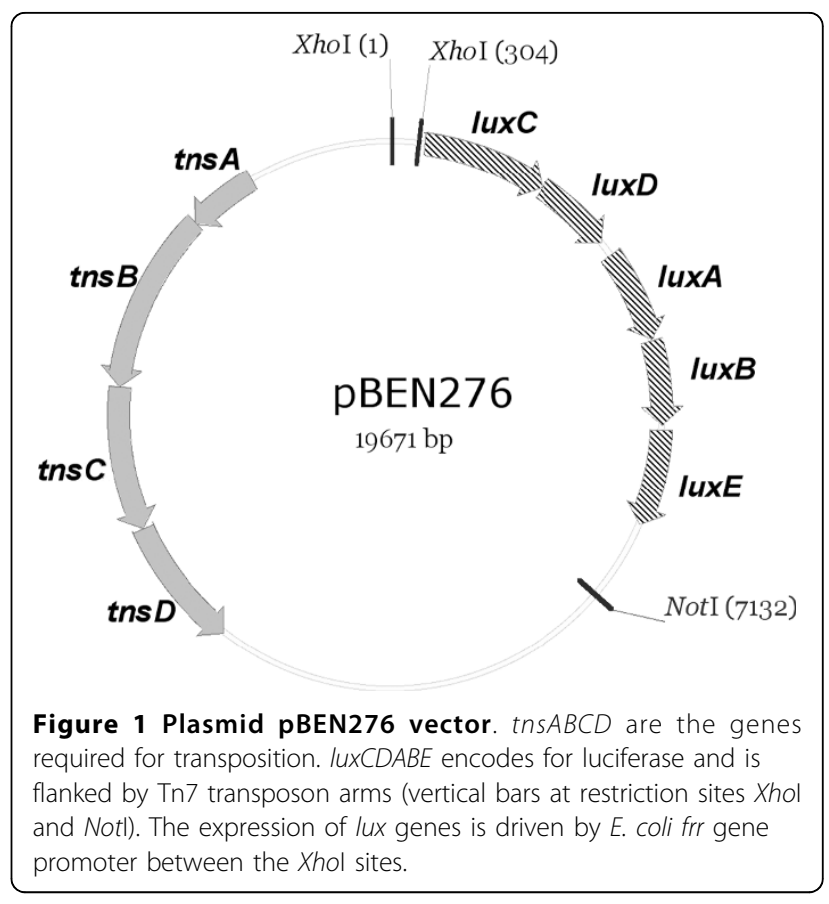

minimum detectable concentration of all eleven serotypes was, in decreasing order $(\mathrm{CFU} / \mathrm{mL}): S$. Kentucky $-8.00 \times$ $10^{4}$; S. Mbandaka - $4.99 \times 10^{4}$; S. Enteritidis - $3.10 \times 10^{4}$; $S$. Schwarzengrund $-2.78 \times 10^{4} ; S$. Montevideo $-1.74 \times$ $10^{4}$; S. Alachua - $1.07 \times 10^{4} ; S$. Typhimurium - $6.72 \times 10^{3}$; $S$. Seftenberg - $6.40 \times 10^{3} ; S$. Heidelberg $-5.28 \times 10^{3}$; $S$. Newport - $4.64 \times 10^{3} ; S$. Braenderup - $4.16 \times 10^{3}$. Minimum detectable numbers of Salmonella isolates expressing bioluminescence from the chromosome were higher compared to minimum detectable numbers of Salmonella isolates expressing plasmid-based bioluminescence [19]. One possible explanation for this difference is a copy number effect; a single copy of the lux operon is inserted into the chromosome with the Tn7 system, while multiple copies of the gene are expressed in plasmid systems. Plasmid pAKlux 1 is a pBBR1 derived plasmid which characteristically has a medium copy number ( 30 copies/cell $)$ [22]. Another possible explanation is due to promoter effect; the frr promoter drives expression of $l u x C D A B E$ in the Tn7 system, and the $l a c Z$ promoter drives expression in the pAKlux1 plasmid system [19]. Our previous work showed bioluminescent Salmonella isolates carrying plasmid pAKlux1 emit, on average, $6.3351 \mathrm{p} / \mathrm{s} / \mathrm{cm}^{2} / \mathrm{sr}$ per CFU [19]; in comparison, Salmonella isolates carrying the lux operon in the chromosome emit, on average, 0.0795 $\mathrm{p} / \mathrm{s} / \mathrm{cm}^{2} / \mathrm{sr}$ per CFU. The intended purpose for this system is to use it as a screening tool for potential pathogen mitigation strategies, and this threshold of detection is sufficient for this purpose. 


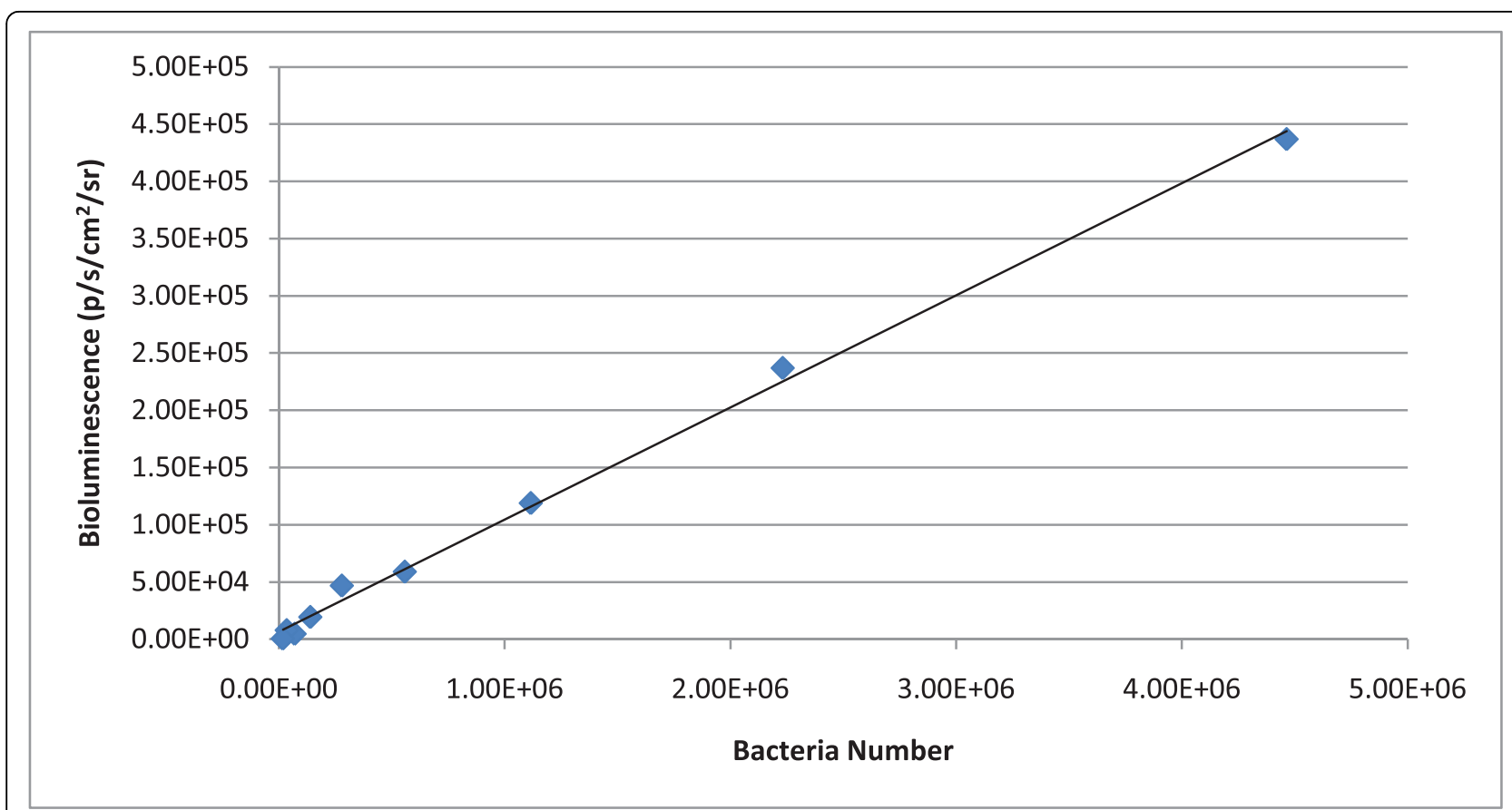

Figure 2 Correlation of bioluminescence against bacterial numbers. Plot and linear regression equation of bioluminescence flux against bacterial numbers for $S$. Montevideo. $r^{2}=0.94, P=<0.0001$.

\section{Transgene stability in the chromosome of Salmonella enterica}

Our group evaluated the stability of the lux operon in the chromosome following transposition by subcloning bioluminescent Salmonella enterica serotypes under non-selective conditions for 14 days at $37^{\circ} \mathrm{C}$. Previous work from our group with plasmid-based bioluminescence expression showed the plasmid was unstable without antibiotic selection. The average half-life of plasmid

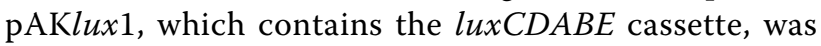
approximately seven days in Salmonella enterica serotypes without antibiotic selection [19]. This current study provides evidence for a 14 day period indicating stability of the lux operon in the chromosome of these Salmonella enterica serotypes with minimal bioluminescent flux (Figure 3). A notable observation was low initial expression of bioluminescence from $S$. Schwarzengrund $\left(10^{5} \mathrm{p} / \mathrm{s} / \mathrm{cm}^{2} / \mathrm{sr}\right)$. This serotype increased bioluminescence expression over the course of the experiment and reached similar levels of the other serotypes at approximately day $10\left(10^{7} \mathrm{p} / \mathrm{s} / \mathrm{cm}^{2} / \mathrm{sr}\right)$. The differences observed for $S$. Schwarzengrund are interesting. It is important to note that the Tn7 transposon system does not insert randomly in the Salmonella chromosome. The Tn7 transposon system is site specific; insertion is only allowed at the $\operatorname{attTn} 7$ site. Therefore, 'luxCDABE mutants' are not possible. Bacterial density values $\left(\mathrm{OD}_{600}\right)$ for $S$. Schwarzengrund were also similar to bacterial density values for the other serotypes. The differences in bioluminescence expression are due to a difference in host serotype background. Determination of the cause of this serotype-specific effect is beyond the scope of the current manuscript. It is of interest that expression of bioluminescence in $S$. Schwarzengrund was also the lowest in the plasmid lux system, pAKlux1, reported previously [19]. These results indicate plasmid pBEN276 can be utilized to construct a stable reporting system within the chromosome of Salmonella enterica serotypes for use in extended in-vitro and in-vivo trials.

\section{Assessment of bioluminescent assay at various temperatures}

Genes luxCDABE encode bacterial luciferase which catalyzes the oxidation of reduced flavin mononucleotide $\left(\mathrm{FMNH}_{2}\right)$ and a long chain aliphatic aldehyde in the presence of $\mathrm{O}_{2}$ to produce flavin mononucleotide (FMN) and acid with light emission. Because $\mathrm{FMNH}_{2}$ production is dependent on a functional electron transport chain, only metabolically active bacteria emit light [23]. Thus, BLI provides a sensitive real-time measurement of the effects of various chemical, biological and physical stimuli on bacterial metabolism [24]. We utilized our bioluminescent Salmonella enterica serotypes to validate our model under a temperature range that bacteria in food products are commonly exposed to (host to ambient to refrigeration). Therefore we investigated the relationship between cellular metabolic activity, characterized by bacterial light production, and 


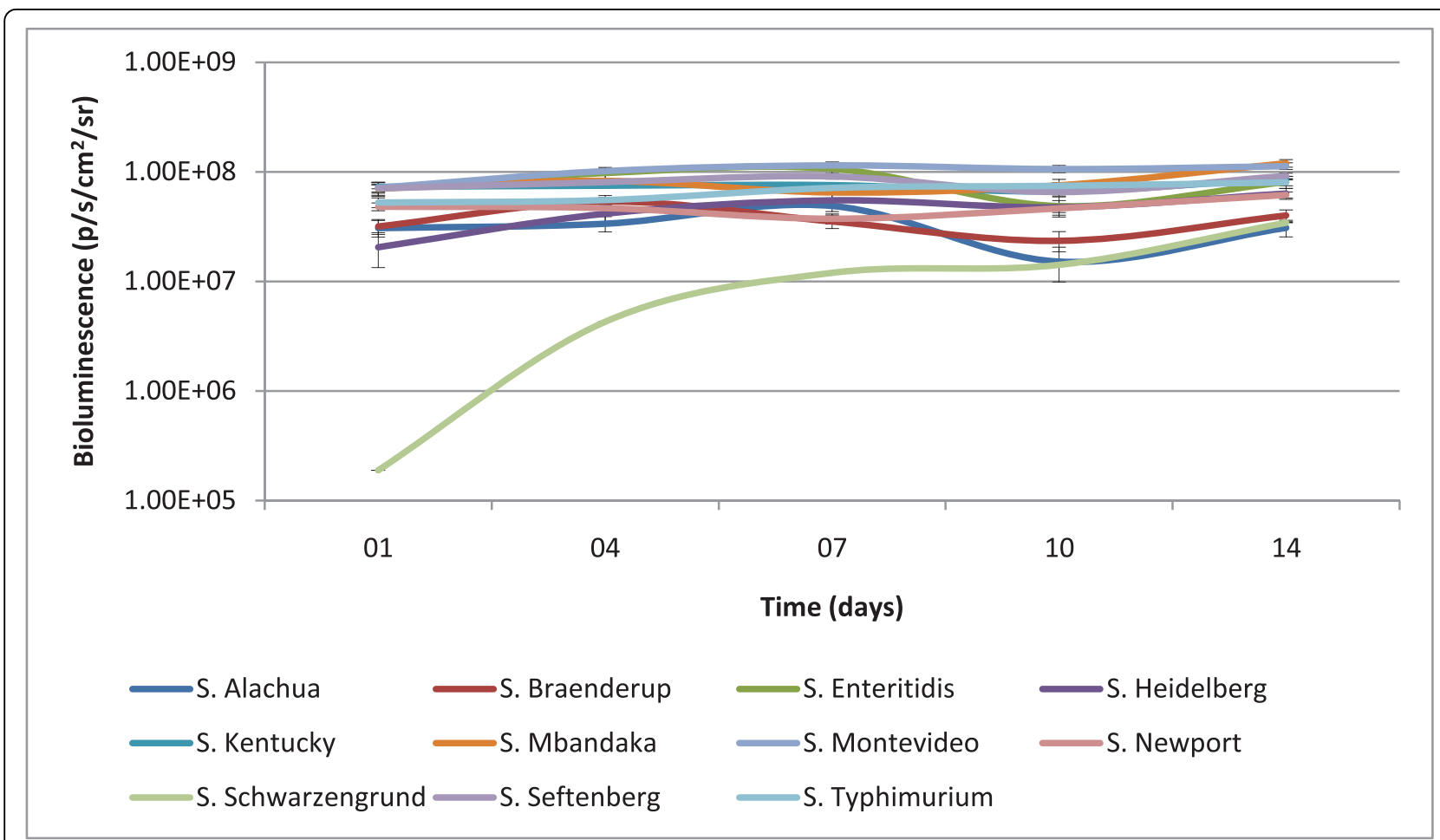

Figure 3 Stability of transgene in chromosome of Salmonella enterica serotypes. Salmonella enterica isolates carrying transgene luxCDABE in their chromosome were subcloned under non-selective conditions for 14 days. Bioluminescence was quantified approximately every 3 days and normalized with bacterial density $\left(\mathrm{OD}_{600}\right)$.

temperature variation. The temperatures selected were $37^{\circ} \mathrm{C}, 25^{\circ} \mathrm{C}$ and $4^{\circ} \mathrm{C}$.

Mesophiles, such as Salmonella grow best in moderate temperatures $\left(15-40^{\circ} \mathrm{C}\right)$ with normal enzymatic activity. In this experiment luciferase reaction within Salmonella was monitored. At $37^{\circ} \mathrm{C}$ and $25^{\circ} \mathrm{C}$ BLI measurements were consistent within the replicates of the different serotypes. However, a change in temperature will have an impact on enzyme kinetics. Decreasing temperature, to $4^{\circ} \mathrm{C}$, will slow molecular motion and inhibit the luciferase reaction. Decreasing temperature will also decrease the rate of metabolism, which translates to decreased concentration of substrate, $\mathrm{FMNH}_{2}$, available for the luciferase reaction. At $4^{\circ} \mathrm{C}$ we observed an expected reduction in bioluminescent signal compared to readings at the two higher temperatures, $37^{\circ} \mathrm{C}$ and $25^{\circ} \mathrm{C}$ (data not shown). However, over the time required (approximately $1 \mathrm{~min}$ ) to complete $\mathrm{BLI}$ measurements at $25^{\circ} \mathrm{C}$ we observed a rapid increase in the bioluminescent signal between the first and the last wells read. We found that luciferase activity is restored shortly after removal from refrigeration temperature, so temperature effect is minimal after introduction to ambient temperatures $\left(\geq 25^{\circ} \mathrm{C}\right)$. These results were consistent and validated that our reporting system using bioluminescent Salmonella can be successfully applied to monitor within a temperature range that bacteria in food products are commonly exposed to.

The stage on our luminometer has adjustable temperature with the lowest temperature setting being $25^{\circ} \mathrm{C}$. Future work will include the development of a mechanism for maintaining plates at refrigeration temperatures while on the reading stage of the instrument to overcome this limitation.

\section{Development of chicken skin assay for real-time monitoring of bioluminescent Salmonella enterica} Salmonella presents a major problem for the poultry industry due to its persistence during the processing of chicken carcasses and few options exist that completely eliminate the bacteria from the chicken carcasses besides proper cooking. The physiological mechanisms which allow the bacteria to persist throughout processing of the chicken carcass are largely unknown and until the mechanics of attachment of the bacteria are more comprehensively understood, Salmonella may well remain a food safety concern.

We have developed a model, which uses a real-time stable reporting system incorporating our bioluminescent tagged Salmonella enterica serotypes, which can be used to evaluate various pathogenic mitigation strategies. Further, this model may eventually aid in the 
understanding of how these serotypes are able to survive the processing continuum. We performed this experiment to demonstrate the potential value of this model as a screening tool by evaluating the performance of our bioluminescent Salmonella on chicken skin sections at two temperatures in an aqueous environment. We selected $S$. Mbandaka and $S$. Montevideo for this skin attachment experiment based on the consistent bioluminescence expression we observed within these serotypes (Figure 3). Individual aqueous solutions, each containing a Salmonella enterica serotype, were prepared and introduced to chicken skin according to protocol (described below). Separate plates (24-well) containing replicates of each serotype were placed on a rotating stage at $4^{\circ} \mathrm{C}$ and $25^{\circ} \mathrm{C}$ for $2 \mathrm{~h}$. Immediately following this step, bioluminescent imaging was collected after a five minute interval at $37^{\circ} \mathrm{C}$ for both serotypes and is reported (Figure 4). Bioluminescent monitoring demonstrated the ability to quantify bacteria numbers on chicken skin following cold and warm washes. Our previous work showed washing with $25^{\circ} \mathrm{C}$ water suppressed the reproduction of Salmonella on chicken skin likely through the physical removal of bacteria [19]. Given that Salmonella is a mesophile, refrigeration temperatures further limit bacterial growth and the bacteria become metabolically static. Bioluminescent values, confirming bacteria numbers, at post-wash $\left(4^{\circ} \mathrm{C}\right)$ were not shown to be significantly different compared to pre-wash values for both serotypes $(P \geq 0.25)$. Bioluminescent values at post-wash $\left(25^{\circ} \mathrm{C}\right)$ were greater compared to pre-wash values but the difference was not shown to be significantly different $(P \geq 0.125)$. The increase in bioluminescence following the $25^{\circ} \mathrm{C}$ wash period is due to increased bacteria growth under favorable metabolic conditions (temperature) and nutrients provided by the chicken skin in solution. With our model we were able to quantify a change in bacteria number by monitoring bioluminescence following treatment.

These results provide evidence that our model may serve as an accurate and efficient means for in-vitro evaluation of the efficacy of pathogen mitigation strategies, i.e. antimicrobial compounds (AMC) and processing parameters, that may be utilized in the poultry processing industry to control Salmonella enterica. Future work utilizing our lux reporter system in our chicken skin model will feature an extended time course to better reflect the duration of exposure to conditions bacteria might be subjected to in the poultry processing environment.

\section{Conclusions}

Our work demonstrates a novel, real-time monitoring system for Salmonella enterica serotypes that is stable and has potential use for in in vivo and in vitro trials.
Our results show the efficiency of plasmid pBEN276 to confer bioluminescence to eleven wild-type Salmonella enterica isolates by inserting the $\operatorname{lux} C D A B E$ operon into the $\operatorname{att} \operatorname{Tn} 7$ site on the chromosome. Chromosomal insertion of the gene is significant in that external antibiotic pressure is not required for perpetuation of the luxCDABE cassette. This system has the potential to eventually be utilized for the evaluation of potential pathogen mitigation strategies upon Salmonella under different environmental conditions over extended time courses, which was not previously possible due to limitations of plasmid-based reporter systems.

Detection was successful following metabolic inactivity due to refrigeration temperatures and results provide support for application of our model in trials simulating processing plant environmental conditions. Future experiments are planned using this system to evaluate the efficacy of various AMCs. We expect this research may provide a foundation for future work to understand the mechanism of attachment of Salmonella to chicken skin and its ability to persist during the poultry processing continuum.

\section{Methods}

\section{Bacterial serotypes and growth media}

As part of a previous study, Salmonella enterica isolates from five different sites along the broiler production continuum (day one placement, end of growout, arrival at the plant, pre-chill tank, and post-chill tank) were cataloged [25]. In the current study, 11 Salmonella enterica serotypes ( $S$. Alachua, S. Braenderup, S. Enteritidis, $S$. Heidelberg, $S$. Kentucky, S. Mbandaka, S. Montevideo, S. Newport, S. Schwarzengrund, S. Seftenberg, $S$. Typhimurium) were selected. Salmonella enterica serotypes were cultured using Luria-Bertani broth and agar plates at $37^{\circ} \mathrm{C}$. Ampicillin $\left(100 \mu \mathrm{g} \mathrm{mL} \mathrm{m}^{-1}\right)$ and was used for selection and $0.1 \%$ arabinose was used for transposition induction.

\section{Construction of plasmid pBEN276}

The luxCDABE operon was amplified from the genome of Photorhabdus luminescens using primers PG131 (GATGCTACCTCGAGGTACAACCAGTTTGCAAGATG) and PG132 (TACGCTCAGGATCCGAATTCACTCCCTTG CCATC) and cloned in pCR2.1 (Invitrogen) to yield plasmid pBEN139. Primers PG131 and PG132 were added to include XhoI and BamHI restriction sites. $A$ XhoI-BamHI restriction fragment from plasmid pBEN139 carrying luxCDABE was subcloned into plasmid pBEN129, a derivative of plasmid pACYC184 [26] containing XhoI and BamHI sites, yielding plasmid pBEN135. A XhoI-NotI fragment from plasmid pBEN135 carrying the luxCDABE operon was subcloned into plasmid pGRG25 [20] to give plasmid 


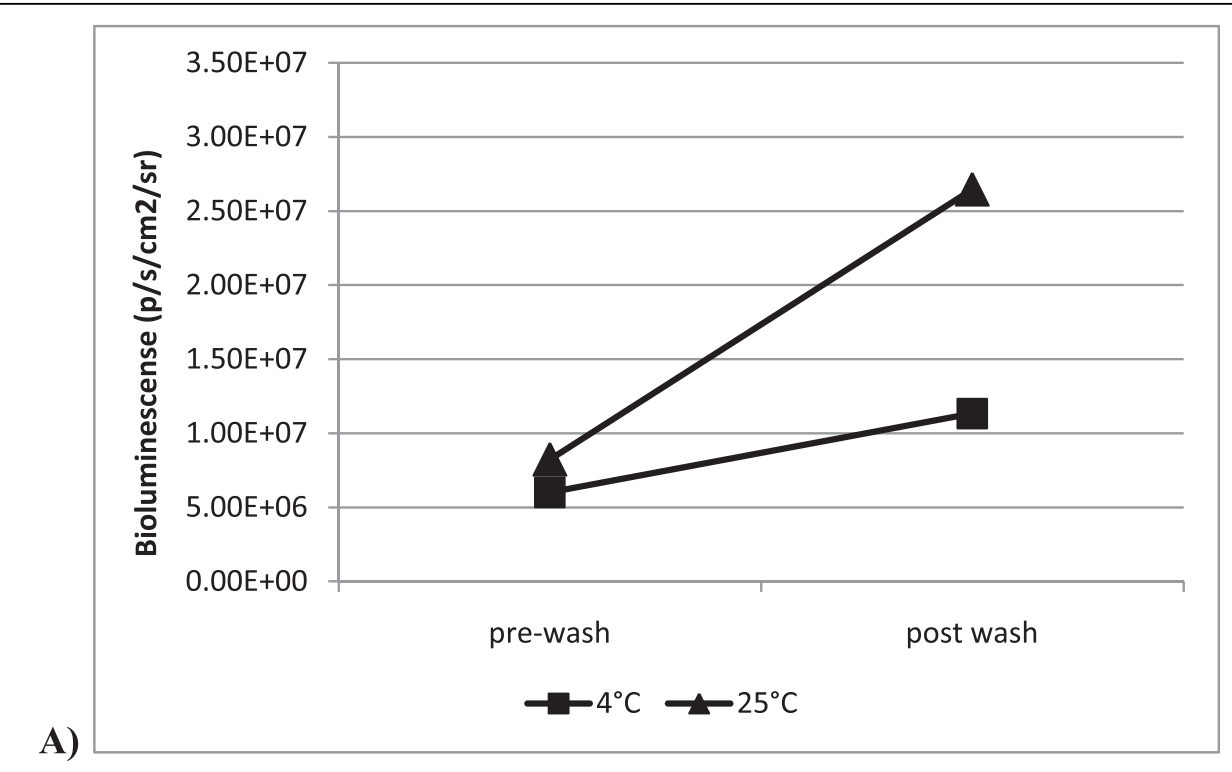

A)

B)

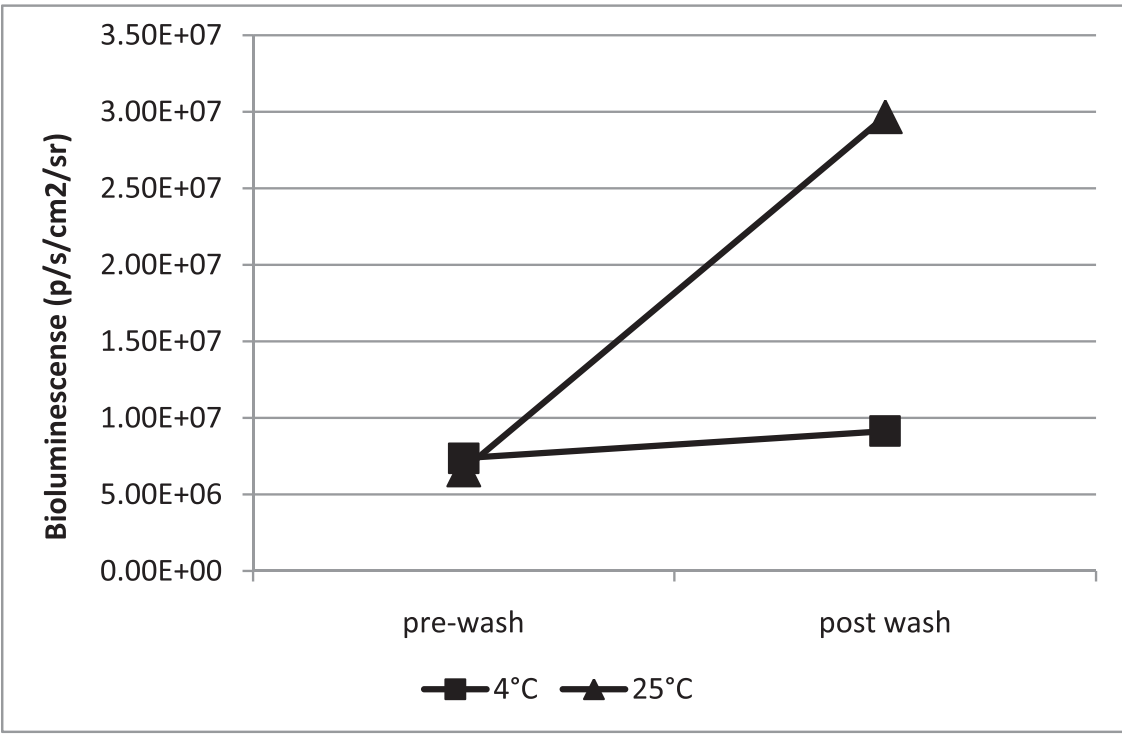

Figure 4 Monitoring bacteria number following $25^{\circ} \mathrm{C}$ and $4^{\circ} \mathrm{C}$ water washes. Bioluminescence quantified at $37^{\circ} \mathrm{C}$ before and after water washes at $4^{\circ} \mathrm{C}$ and $25^{\circ} \mathrm{C}$. A) S. Mbandaka. B) S. Montevideo.

pBEN275. The promoter of the housekeeping gene frr [27] was amplified from the E. coli K-12 MG1655 genome using PG209 (GTCTGACTCGAGGAATTCTTCCCGTGATGGATAAATAAG) and PG210 (CATCACTCGAGGTTACGAATCCTTGAAAACTTG) primers and cloned into the $X h o I$ site of plasmid pBEN275 to give plasmid pBEN276.

Insertion of lux genes into the chromosome of Salmonella enterica

Bioluminescence was established in the chromosome of the Salmonella enterica serotypes using plasmid pBEN276. The serotypes were grown to logarithmic phase $\left(\mathrm{OD}_{600}\right.$ 0.6-0.8), washed with $15 \%$ cold glycerol solution four times to make electrocompetent, and stored at $-80^{\circ} \mathrm{C}$. The serotypes were transformed with plasmid pBEN276 by electroporation using a Gene Pulser II system (Bio-Rad). Optimal electroporation conditions for $S$. Alachua, S. Heidelberg, S. Kentucky, $S$. Mbandaka, $S$. Newport and $S$. Seftenberg were $2.5 \mathrm{kV}$, $25 \mu \mathrm{F}$ and $400 \Omega$, and optimal conditions for $S$. Braenderup, $S$. Enteritidis, $S$. Montevideo, $S$. Schwarzengrund and $S$. Typhimurium were $1.8 \mathrm{kV}, 25 \mu \mathrm{F}$ and $600 \Omega$. Bacteria were recovered for $1 \mathrm{~h}$ at $30^{\circ} \mathrm{C}$ in SOC media and then spread on LB plates with ampicillin and placed in an incubator at $30^{\circ} \mathrm{C}$ for approximately $16 \mathrm{~h}$. Ampicillin 
resistant colonies were picked and cultured in LB broth with arabinose at $30^{\circ} \mathrm{C}$ for approximately $16 \mathrm{~h}$ to induce transposition. The cultures were streaked on LB agar and placed in an incubator at $42^{\circ} \mathrm{C}$ for approximately 16 $\mathrm{h}$ to cure the plasmid. Ten individual colonies were picked from this plate and cultured in LB broth at $42^{\circ} \mathrm{C}$ for approximately $16 \mathrm{~h}$. Bioluminescent colonies were detected using a Chemilmager 5500 imaging system with AlphaEaseFC software (Alpha Innotech) or an IVIS Imaging System 100 Series with Living Image Software v2.50 (Xenogen). Bioluminescent cultures were subcloned in LB broth with ampicillin and placed in an incubator at $30^{\circ} \mathrm{C}$ for approximately $16 \mathrm{~h}$. No visual evidence of growth confirmed absence of the plasmid.

\section{Characterizing the bioluminescent properties of Salmonella enterica serotypes}

The bioluminescent Salmonella enterica serotypes were grown overnight in LB broth to reach stationary phase, and bacterial density value $\left(\mathrm{OD}_{600}\right)$ of each serotype was determined in a 96-well clear-bottomed black cell culture plate (Costar) using ThermoMax spectrometer (Molecular Devices). Following bacterial density measurements, four separate dilution series were prepared for each serotype in 96-well clear-bottomed black cell culture plates. In each plate, the first four columns contained 10 fold dilutions $\left(1.00 \times 10^{0}\right.$ to $\left.1.00 \times 10^{-3}\right)$, while the remaining eight wells contained doubling dilutions $\left(5.00 \times 10^{-4}, 2.50 \times 10^{-4}, 1.25 \times 10^{-4}, 6.25 \times 10^{-4}\right.$, $3.13 \times 10^{-5}, 1.56 \times 10^{-5}, 7.81 \times 10^{-6}, 3.91 \times 10^{-6}, 1.95 \times$ $\left.10^{-6}, 9.77 \times 10^{-7}, 4.88 \times 10^{-7}, 2.44 \times 10^{-7}\right)$. Bioluminescence was measured for $10 \mathrm{~s}$ of exposure using an IVIS Imaging System 100 Series, and bioluminescence was quantified using Living Image software v2.50. The last dilution of each series was spread on LB agar to determine the number of viable bacteria. The linear relationship between population densities and bioluminescence was determined by plotting bioluminescence against bacterial density as determined by plate counts and by linear regression analysis (PROC REG, SAS for Windows v9.2, SAS Institute Inc.). The minimum detectible number for each serotype was determined using the number of bacteria present in the last dilution that had detectable bioluminescence. Colony counts were also used to calculate the theoretical amount of bioluminescence produced from $1 \mathrm{CFU}$ for each serotype.

\section{Transgene stability in the chromosome of Salmonella enterica}

Transgene stability following insertion by plasmid pBEN276 in the eleven Salmonella enterica serotypes was analyzed by subcloning these bioluminescent Salmonella enterica serotypes in non selective LB broth every $24 \mathrm{~h}$ for a period of fourteen days. Technical replicates for each serotype were made in quadruplicate. For each passage, the previous culture was subcloned $1 / 10$ the volume into new $300 \mu \mathrm{L}$ cultures of LB broth in 96-well clear-bottomed black cell culture plates. Bacterial density and bioluminescence was measured at 12 $h$ of growth at approximately every 3 days. Bioluminescence was measured using an IVIS Imaging System for $15 \mathrm{~s}$ of exposure and normalized by dividing total flux of bioluminescence by the corresponding bacterial density value. The average normalized bioluminescence for each serotype and passage was determined, which revealed the ability of each serotype to maintain the lux operon in its chromosome without antibiotic selection.

\section{Assessment of bioluminescent assay at various temperatures}

An experimental model was established to investigate the relationship between temperature variation and metabolic activity, characterized by bioluminescence expression. Bioluminescence and bacterial density were measured using the LMax luminometer (1 s exposure time) and the Spectramax Plus 384 spectrophotometer (Molecular Devices), respectively. Cultures of bioluminescent Salmonella enterica serotypes were grown overnight $(\sim 16 \mathrm{~h})$ to reach stationary phase and were diluted 10 fold with LB broth and $200 \mu \mathrm{L}$ of the diluted bacteria suspension was inoculated into a 96-well clear-bottomed black cell culture plate and incubated at $37^{\circ} \mathrm{C}$ for $2 \mathrm{~h}$ to reach early log phase. Four technical replicates for each serotype were prepared. The initial bioluminescence and bacterial density reading was collected for the early log phase cultures at $37^{\circ} \mathrm{C}$. Next, the plate incubated for 10 min at $25^{\circ} \mathrm{C}$, and bioluminescence and bacterial density readings were measured. Then, the plate was transferred to $4^{\circ} \mathrm{C}$ and stayed at this temperature for $2 \mathrm{~h}$, interrupted every $30 \mathrm{~min}$ to measure bioluminescence and bacterial density.

\section{Development of chicken skin assay for real-time monitoring of bioluminescent Salmonella enterica} Overnight cultures of bioluminescent Salmonella enterica serotypes, $S$. Mbandaka and $S$. Montevideo, were prepared in replicates in quadruplicate. Each individual solution was diluted to approximately $1 \times 10^{6} \mathrm{CFU} / \mathrm{mL}$ in distilled water, and $1 \mathrm{~mL}$ of the bacteria suspension was added to $8 \mathrm{~mm}$ circular chicken skin sections in 24-well polystyrene clear-bottomed black tissue culture plates (Wallac) as described previously [19]. Plates were incubated at room temperature $\left(25^{\circ} \mathrm{C}\right)$ for $1 \mathrm{~h}$ to allow bacteria to attach to the skin. Following incubation, the suspension was vacuumed from each well, and skin sections were gently washed with distilled water and vacuumed to remove unattached bacteria. This washing process was repeated once more. 
After removal of excess solution, initial bioluminescence on skin sections was quantified for $15 \mathrm{~s}$ of exposure using the IVIS imaging system. One $\mathrm{mL}$ of $4^{\circ} \mathrm{C}$ distilled water was added to each well of the appropriate plate for each serotype. The other plate for each serotype received one $\mathrm{mL}$ of $25^{\circ} \mathrm{C}$ distilled water. The plate that received $4^{\circ} \mathrm{C}$ distilled water remained at refrigeration temperature $\left(4^{\circ} \mathrm{C}\right)$ for $2 \mathrm{~h}$ on a rotating stage at 200 $\mathrm{rpm}$. The plate that received $25^{\circ} \mathrm{C}$ distilled water remained at room temperature $\left(25^{\circ} \mathrm{C}\right)$ for $2 \mathrm{~h}$ on a rotating stage at $200 \mathrm{rpm}$. At the conclusion of the $2 \mathrm{~h}$ washing period, water was vacuumed from each well, and bioluminescence from bacteria attached to the chicken skin was measured at $37^{\circ} \mathrm{C}$ for $5 \mathrm{~min}$. The total flux of bioluminescence from each well was divided by the corresponding bacterial density value of the original bacterial suspension to normalize bioluminescent flux.

\section{Acknowledgements}

We thank Dr. Alain Givaudan (INRA, Université Montpellier II, Montpellier, FRANCE) for providing us with Photorhabdus luminescens genomic DNA. We acknowledge Dr. Scott Willard and Dr. Peter Ryan for use of the IVIS Living Image System in the MSU Laboratory for Organismal and Cellular Imaging. This study was funded by the U.S. Department of Agriculture, Agricultural Research Service (agreement no 321956-182070-027000-371290).

\section{Author details}

'Department of Pathobiology and Population Medicine, College of Veterinary Medicine, Mississippi State University, Mississippi State, MS 39762, USA. ${ }^{2}$ Department of Basic Sciences, College of Veterinary Medicine, Mississippi State University, Mississippi State, MS 39762, USA. ${ }^{3}$ Institute for Digital Biology, Mississippi State University, Mississippi State, MS 39762, USA ${ }^{4}$ INRA, UR 1282 Infectiologie Animale et Santé Publique, Laboratoire de Pathogénie Bactérienne, Nouzilly, France.

\section{Authors' contributions}

$\mathrm{RB}$ and RW isolated the Salmonella strains. PG constructed the PBEN276 plasmid. AK, RB, KH, and $M L$ designed the bacteriological and genetic studies. AK, RW and $\mathrm{KH}$ performed the experiments and data analyses. AK, $\mathrm{RB}, \mathrm{KH}, \mathrm{ML}, \mathrm{RW}$ and $\mathrm{PG}$ drafted the manuscript. All authors read and approved the final manuscript.

Received: 18 December 2009 Accepted: 23 July 2010

Published: 23 July 2010

\section{References}

1. Ohl ME, Miller SI: Salmonella: a model for bacterial pathogenesis. Annu Rev Med 2001, 52:259-274.

2. Ly KT, Casanova JE: Mechanisms of Salmonella entry into host cells. Cell Microbiol 2007, 9:2103-2111.

3. Sarlin LL, Barnhart ET, Caldwell DJ, Moore RW, Byrd JA, Caldwell DY, Corrier DE, Deloach JR, Hargis BM: Evaluation of alternative sampling methods for Salmonella critical control point determination at broiler processing. Poult Sci 1998, 77:1253-1257.

4. Lillard HS: Incidence and recovery of Salmonellae and other bacteria from commercially processed poultry carcasses at selected pre- and post-evisceration steps. J Food Prot 1989, 52:88-91.

5. Lillard HS: The impact with commercial processing procedures on the bacterial contamination and cross-contamination of broiler carcasses. J Food Prot 1990, 53:202-204.

6. Lillard HS: Bacterial cell characteristics and conditions influencing Salmonella adhesion to poultry skin. J Food Prot 1985, 48:803-807.
7. Lillard HS: Distribution of "attached" Salmonella typhimurium cells between poultry skin and a surface film following water immersion. J Food Prot 1986, 49:449-454.

8. McMeekin TA, Thomas CJ: Retention of bacteria on chicken skin after immersion in bacterial suspensions. J Food Prot 1978, 48:939-943.

9. Lillard HS: Factors affecting the persistence of Salmonella during the processing of poultry. J Food Prot 1989, 52:829-832.

10. Kinsella KJ, Rowe TA, Blair IS, McDowell DA, Sheridan JJ: The influence of attachment to beef surfaces on the survival of cells of Salmonella enterica serovar Typhimurium DT104, at different $\mathrm{a}(\mathrm{w})$ values and at low storage temperatures. Food Microbiol 2007, 24:786-793.

11. Li Y, Slavik MF, Walker JT, Xiong H: Pre-chill spray of chicken carcasses to reduce Salmonella Typhimurium. J Food Sci 1997, 62:605-607.

12. Mullerat J, Sheldon BW, Klapes NA: Inactivation of Salmonella species and other food-borne pathogens with Salmide, a sodium chlorite-based oxyhalogen disinfectant. J Food Prot 1995, 58:535-540.

13. Rathgeber BM, Waldroup AL: Antibacterial activity of a sodium acid pyrophosphate product in chiller water against bacteria on broiler carcasses. J Food Prot 1995, 58:530-534.

14. Basti AA, Razavilar V: Growth response and modeling of the effects of selected factors on the time-to-detection and probability of growth initiation of Salmonella Typhimurium. Food Microbiology 2004, 21:431-438.

15. Contag PR, Olomu IN, Stevenson DK, Contag CH: Bioluminescent indicators in living mammals. Nat Med 1998, 4:245-247.

16. Contag $\mathrm{CH}$, Contag PR, Mullins JI, Spilman SD, Stevenson DK, Benaron DA Photonic detection of bacterial pathogens in living hosts. Mol Microbiol 1995, 18:593-603.

17. Contag $\mathrm{CH}$, Bachmann $\mathrm{MH}$ : Advances in in vivo bioluminescence imaging of gene expression. Annu Rev Biomed Eng 2002, 4:235-260.

18. Karsi A, Menanteau-Ledouble S, Lawrence ML: Development of bioluminescent Edwardsiella ictaluri for noninvasive disease monitoring FEMS Microbiol Lett 2006, 260:216-223.

19. Karsi A, Howe K, Kirkpatrick TB, Wills R, Bailey RH, Lawrence ML: Development of bioluminescent Salmonella strains for use in food safety. BMC Microbiol 2008, 8:10.

20. McKenzie GJ, Craig NL: Fast, easy and efficient: site-specific insertion of transgenes into enterobacterial chromosomes using Tn7 without need for selection of the insertion event. BMC Microbiol 2006, 6:39.

21. Waddell CS, Craig NL: Tn7 transposition: recognition of the attTn7 target sequence. Proc Natl Acad Sci USA 1989, 86:3958-3962.

22. Lynch MD, Gill RT: Broad host range vectors for stable genomic library construction. Biotechnol Bioeng 2006, 94:151-158.

23. Alloush HM, Lewis RJ, Salisbury VC: Bacterial bioluminescent biosensors: Applications in food and environmental monitoring. Analytical Letters 2006, 39:1517-1526.

24. Billard P, DuBow MS: Bioluminescence-based assays for detection and characterization of bacteria and chemicals in clinical laboratories. Clin Biochem 1998, 31:1-14.

25. Volkova W, Bailey RH, Rybolt ML, Dazo-Galarneau K, Hubbard SA, Magee D, Byrd JA, Wills RW: Inter-relationships of Salmonella Status of Flock and Grow-Out Environment at Sequential Segments in Broiler Production and Processing. Zoonoses and Public Health 2009.

26. Chang AC, Cohen SN: Construction and characterization of amplifiable multicopy DNA cloning vehicles derived from the P15A cryptic miniplasmid. J Bacteriol 1978, 134:1141-1156.

27. Liu M, Durfee T, Cabrera JE, Zhao K, Jin DJ, Blattner FR: Global Transcriptional Programs Reveal a Carbon Source Foraging Strategy by Escherichia coli. Journal of Biological Chemistry 2005, 208:15921-15927.

doi:10.1186/1471-2180-10-197

Cite this article as: Howe et al:: Development of stable reporter system cloning luxCDABE genes into chromosome of Salmonella enterica serotypes using Tn7 transposon. BMC Microbiology 2010 10:197. 